\title{
Typologie de l'artiste sandien dans les Lettres d'un voyageur (1834-1837). Écriture et pouvoir
}

Nicole Mozet

\section{OpenEdition}

\section{Journals}

Édition électronique

URL : http://journals.openedition.org/recherchestravaux/193

DOI : 10.4000/recherchestravaux.193

ISSN : 1969-6434

Éditeur

UGA Éditions/Université Grenoble Alpes

\section{Édition imprimée}

Date de publication : 15 avril 2007

Pagination : 133-140

ISBN : 978-2-84310-107-7

ISSN : 0151-1874

Référence électronique

Nicole Mozet, « Typologie de l'artiste sandien dans les Lettres d'un voyageur (1834-1837). Écriture et pouvoir », Recherches \& Travaux [En ligne], 70 | 2007, mis en ligne le 02 décembre 2008, consulté le 08 septembre 2020. URL : http://journals.openedition.org/recherchestravaux/193 ; DOI : https://doi.org/ 10.4000/recherchestravaux.193 
Nicole MozeT

Université Denis-Diderot - Paris 7

\section{Typologie de l'artiste sandien dans les Lettres d'un voyageur (1834-1837) Écriture et pouvoir}

Poètes, musiciens, peintres et sculpteurs, philosophes, prosateurs, orateurs, "grands écrivains " et amateurs, toute la constellation de ceux qui peuvent prétendre au titre d'" artiste " est présente dans les Lettres d'un voyageur. Le Voyageur ne manque jamais de se situer dans un coin du tableau, jamais tout à fait le même, jusqu'au fameux " il ne nous reste plus que George Sand et compagnie " (p. 942 ; p. 318 $)$ de la "Lettre à M. Nisard ", magnifique de mépris et de maîtrise de soi. Ce très ironique passage du dernier paragraphe sert de clausule à l'ensemble du volume :

Il me reste à vous remercier, monsieur, pour les bons conseils que vous m'avez donnés. Je m’accuse, je le répète ; car si vous ne m’avez pas toujours bien compris, c'est ma faute et non la vôtre. L'homme qui contemple une bataille du haut de la montagne juge mieux des fautes et des pertes des armées que celui qui marche dans la poussière et l'enivrement du combat. Ainsi le critique sans passion en sait plus long sur l'artiste bouillant et sur son travail que l'artiste luimême. (P. $943 ;$ p. 318.)

La lettre XII tourne à sa manière la page de ce que l'on appelle déjà, depuis le Stendhal de I8I7, le classicisme. C'est pourquoi, essayant de penser la part de pouvoir symbolique que Sand s'attribuait en tant qu'écrivain, je commence par cette formule provocante, car elle est célèbre mais peut être interprétée de bien diverses façons. Manifeste-t-elle de la modestie ou de l'orgueil ? Qui ne fait pas partie de cette « compagnie » ? Les grands noms du

I. Selon la convention que nous adoptons pour ce volume: G. Sand, Lettres d'un voyageur, pages de l'édition de G. Lubin puis pages de l'édition de H. Bonnet. 
passé assurément, comme Aristophane, Térence ou Molière, qui sont mentionnés au début du même paragraphe avant qu’il soit dit que «la plume de tels écrivains est à jamais brisée " (p. 942 ; p. 318). Mais est-ce que tous les contemporains, artistes et/ou écrivains, appartiennent à cette " compagnie » dont George Sand se fait fièrement le chef de file? Sans doute, mais sûrement pas au même titre ni à la même place. La distribution des rôles repose pour l'essentiel sur une opposition entre la foi qui transforme les paroles en promesses, et les analyses des traces, toujours sous le signe du doute:

Le doute est le mal de notre âge, comme le choléra. Mais bénéfique comme toutes les crises où Dieu pousse l'intelligence humaine, il est le précurseur de la santé morale, de la foi. Le doute est né de l'examen. Il est le fils malade et fiévreux d'une puissante mère, la liberté. (Préface ; p. 648 ; p. 40.)

Le balancement entre transcendance et immanence est un mouvement propre à tout le romantisme, ce qui devrait empêcher d'appliquer aux textes sandiens le terme d' « idéalisme ». J'y reviendrai en conclusion en reprenant le texte de Zola.

Ce que je voudrais ici mettre en lumière, c'est la manière sandienne de prendre le pouvoir, envers et contre tous, qu'il s'agisse des gendarmes de Louis-Philippe, du pape ou de Talleyrand, de Musset, de Canova, de Michel de Bourges ou de Liszt. Dans le passage terminal de la Lettre à Nisard que j’ai cité plus haut, Sand se décrit comme "celui qui marche dans la poussière et l'enivrement du combat ". Quel que soit l'" adversaire ", après beaucoup de feintes et de dérobades, elle s'arrange pour gagner.

Tout au long de la genèse des Lettres d'un voyageur, la guerre que Sand a livrée contre elle-même et les autres a duré trois ans : de I834 à I837. Elle s'est effectuée sur deux fronts, celui du privé et celui du public, comme j'ai tenté de le montrer dans ma préface au tome $\mathrm{X}$ de l'édition d'Histoire de ma vie ${ }^{2}$, qui porte essentiellement sur le rôle déterminant qu'a joué pour Sand le procès, en I835 à Paris, des insurgés de Lyon : c'est parce qu'elle s'est confrontée directement, physiquement, à la chose publique, politique et judiciaire, qu'elle a eu ensuite le courage d'affronter son mari et d'entamer un procès en séparation. C'est avec la même audace qu'elle affronte Nisard, qui l'avait bassement attaquée sur sa vie privée, alors que Casimir venait de faire appel et que son procès était encore en cours. La réponse cinglante au critique paraît dans la Revue de Paris du 29 mai I836 et le procès en séparation ne sera clos que le 29 juillet. Ainsi, je pense que Sand n'a pu continuer à écrire que grâce

2. N. Mozet, "Politique et divorce ", préface à G. Sand, Histoire de ma vie, vol. X, SaintCyr-sur-Loire, Christian Pirot éditeur, 2003. 
à son séjour à Venise, qui lui a rendu et Paris et Nohant. C'est au demeurant la ligne générale du volume des Lettres d'un voyageur, qui va de Musset à Nisard. Chaque lettre est une bataille. L'ordre des lettres selon l'organisation du volume m'a paru plus pertinent pour mon propos que celui des parutions en revue, surtout à cause de la place centrale qui est donnée à la lettre VI (« À Éverard»). La logique de la démarche initiatique se manifeste plus clairement dans le recueil que dans les revues, grâce à la mise en série, qui fait récit et se moule tout naturellement sur le modèle de la quête.

Les trois premières lettres sont celles du cycle vénitien. Dans la lettre I, Musset le poète est opposé aux " esprits médiocres et paresseux comme le mien» (p. 652; p. 43), mais il a le malheur d'être possédé par l'" amour de la destruction " (p. 66I ; p. 5I). Il lui manque aussi «le temps d'aimer le repos» (p. 652 ; p. 43). C'est ensuite le médecin, autre figure du pouvoir, qui traite le Voyageur de "chétif individu» (p. 654; p. 44). Ce chétif individu n'en part pas moins seul pour une randonnée à pied dans les montagnes au-dessus de Venise. Il connaît la fatigue et la peur, mais il a vu les montagnes de Canova, il a su se reposer et guérir, et il revient pour repartir aussitôt. Ce nouveau départ, pour un «Tyrol» (p. 678; p. 67) imaginaire et inaccessible, constitue la victoire de la première lettre. C'est pourtant à Venise qu'on se retrouve dans la lettre suivante, celle du rêve, très proche du Corambé d' Histoire de ma vie, la Venise des cafés et du chant de ses gondoliers. La victoire est à nouveau celle du savoir dormir :

Je défie qui que ce soit de m'empêcher de dormir agréablement quand je vois Venise, si appauvrie, si opprimée et si misérable, défier le temps et les hommes de l'empêcher d'être belle et sereine. (P. 704 ; p. 9I.)

Il faut comprendre que c'est contre lui-même que le Voyageur a livré sa première bataille : reprendre pied, se retrouver après la tourmente, ne pas céder aux démons de la folie ni à la tentation du suicide. C'est ce que j'appelle " guérir ». La plainte s'exhale longuement dans la lettre IV, écrite à Nohant ("Je suis un malade qu'il faut plaindre et non contrarier"; p. 736 ; p. I23). Pourtant, c'est dans cette même lettre qu'elle revendique Lélia :

Je suis bien fâché d'avoir écrit ce mauvais livre qu'on appelle Lélia, non pas que je m'en repente : ce livre est l'action la plus hardie et la plus loyale de ma vie, bien que la plus folle et la plus propre à me dégoûter de ce monde à cause des résultats. (P. 754 ; p. 140.)

Formule très sandienne par son ambiguïté, qui permet tous les contresens. En effet, si l'on ne retient que "mauvais livre", on est aux antipodes de ce que Sand pense de son ouvre. Dans les Lettres d'un voyageur comme dans la Correspondance ou les préfaces, la fierté de l'auteur se redresse au moment où 
l'on s'y attend le moins. C'est un type de prise de parole et de pouvoir exactement comparable au " George Sand et compagnie » de la Lettre à Nisard et au « je pars après-demain pour le Tyrol... " (p. 678 ; p. 67) qui ferme en fanfare la première lettre. La lettre $V$, adressée à François Rollinat, clôt cette première partie, plutôt intimiste, du parcours sandien. Tout en conservant une teinte mélancolique, elle est beaucoup plus apaisée : "Je supporte donc la vie, parce que je l'aime [...] » (p. 764 ; p. I49). Cette phrase vient après un long paragraphe sur le suicide et l'horreur de la mort, laquelle représente le seul antidote à la tentation du suicide :

Il est des crises violentes où le suicide devient un besoin, une rage ; c'est une certaine portion du cerveau qui souffre et s'atrophie physiquement. Mais que cette crise passe ; la nature, la robuste nature que Dieu a faite pour durer son temps, étend ses bras désolés et se rattache aux moindres brins d'herbe pour ne pas rouler dans sa fosse. En faisant la vie de l'homme si misérable, la Providence a bien su qu'il fallait donner à l'homme l'horreur de la mort. Et cela est le plus grand, le plus inexplicable des miracles qui concourent à la durée du genre humain ; car quiconque verrait clairement ce qui est, se donnerait la mort. (P. 764; p. I48.)

La métaphore du marcheur se trouve ensuite longuement développée, avec ses chutes, ses redressements et ses rechutes. La fin de la lettre pivote sur une minuscule violette cueillie par le Malgache : "J'y cours. Adieu, Pylade " (p. 779 ; p. 162).

Au milieu de ce cheminement, il ne faut pas s'étonner de voir surgir Michel de Bourges, homme de génie et de pouvoir, grâce à qui Sand va bientôt pouvoir surmonter l'épreuve de la séparation conjugale et venir se placer beaucoup plus franchement sur le devant de la scène. À cette époque de sa vie, Éverard a été le pivot, le démiurge et le passeur. En avril I835, il est à Paris, où Sand ira le rejoindre après l'avoir rencontré pour la première fois à Bourges au début du mois. Michel de Bourges est en effet l'un des avocats qui assurent devant la Chambre des pairs la défense des insurgés lyonnais du " procès monstre ». Les relations entre Sand et Michel de Bourges changeront complètement par la suite, surtout en I848. De celui-ci, George Sand dira tristement à la fin d'Histoire de ma vie, en évoquant 1848 : "J'étais devenue socialiste, Éverard ne l'était plus ${ }^{3}$ ».

La lettre VI, centrale dans l'économie globale des Lettres d'un voyageur, l'est également pour la démonstration que je tente d'élaborer, quoique Éverard ne soit pas un artiste. Mais c'est par rapport à lui, à qui elle reconnaît du " génie », qu'elle définit l'ensemble de la catégorie artiste dans laquelle elle se situe elle-même. La stratégie énonciative est tortueuse, touffue sinon confuse,

3. G. Sand, Histoire de ma vie, édition de G. Lubin, op. cit., vol. II, p. 364. 
et mérite une analyse détaillée. J'y ai repéré huit séquences, quelquefois datées mais toujours typographiquement séparées, fermées par une clausule en forme de pirouette impertinente et solennelle à la fois, comme dans beaucoup d'autres lettres : "Adieu, ô mes enfants ! j'ai été jusqu' ici plus enfant que vous; je m'en vais seul et loin en pèlerinage, pour tâcher de vieillir vite et de réparer le temps perdu » (p. 817 ; p. 197). "Vieillir vite ", cela ne signifie pas cesser d'être artiste, c'est accepter de concilier le désir de sublime et l'engagement dans le monde. On verra dans la lettre VII que cela correspond exactement à la définition sandienne de l'écrivain. Les dernières lignes de la lettre VI, tout en réitérant un départ pour un autre Tyrol, amorcent un constat de réalité irréversible :

Ô verte Bohême ! patrie fantastique des âmes sans ambition et sans entraves, je vais donc te revoir! J'ai erré souvent dans tes montagnes et voltigé sur la cime de tes sapins ; je m'en souviens fort bien, quoique je ne fusse pas encore né parmi les hommes, et mon malheur est venu de n'avoir pu t'oublier en vivant ici. (P. 817 ; p. 198.)

Le texte adressé à Éverard est important à cause de ce qu'il s'y dit du pouvoir et du statut de l'artiste. Étant le contraire et l'ennemi déclaré des artistes, Éverard permet à George Sand de se poser en s'opposant. Déjà la première séquence, bien que formulée sous forme d'hommage, refuse à Éverard, parce qu'hypocrite à ses yeux, le titre de philanthrope. Pour Éverard, décrète le Voyageur, il ne s'agit pas d'aimer mais de régner : "Tu n'aimes pas les hommes, tu n'es pas leur frère, car tu n'es pas leur égal. Tu es une exception parmi eux, tu es né roi » (p. 780 ; p. 164). En face, le Voyageur, "indiscipliné voyou " (loc. cit.), se fait à la fois modeste et sarcastique. La deuxième séquence met face à face les « hommes de bruit » (p. 783 ; p. I66) et les " rêveurs inoffensifs » (loc. cit.), mais affirme en même temps que le statut d'artiste est loin d'être sans danger, au moins lorsque celui-ci est sincère et honnête : «[...] pour moi, je suis de trop bonne foi pour essayer de me réconcilier par un acte d'hypocrisie avec les sévérités que mon irrésolution (courageuse et loyale, j'ose le dire) attire sur moi " (p. 786 ; p. I69). L'opposition entre la "vertu ", nécessaire aux hommes d'idées et l' « honnêteté » des artistes est reprise dans la troisième séquence, mais avec des revendications de plus en plus précises pour la liberté de l'artiste, et en particulier le droit à la passion amoureuse. La séquence se termine sur cette phrase ironique : "Règne, amour, règne en attendant que la vertu et la république te coupent les ailes» (p. $794 ;$ p. 176).

4. Je souligne parce que c'est le même mot qui est employé pour refuser la prétendue «philanthropie " d'Éverard. 
Dans les deux séquences suivantes, le voyageur plaint le solitaire et l'invite à se laisser consoler par ses amis :

Toi, tu es entré dans ton agonie le jour où tu es né, et le sceau de la douleur t’avait marqué au front dans le sein de ta mère. Viens, nous respecterons ta peine et nous tâcherons d'en alléger le poids. (P. 796 ; p. I78.)

La cinquième séquence est un hymne à l'ami Néraud, dit le Malgache, le botaniste. Avec lui s'engouffrent dans le texte toutes les couleurs, les chants et les odeurs de la nature, mais aussi une profession de foi républicaine, venue du cœur et donc naturelle elle aussi à sa manière :

Quoique né dans le camp opposé, j'avais toujours eu l'âme républicaine, et je l'avais d'autant plus alors que j'étais plus jeune et plus illusionnable. Il me sut un gré extrême d'appartenir à ces types d'hommes obstinés sur lesquels les préjugés de l'éducation ne peuvent rien, et il me déclara qu'il ne me manquait, pour obtenir sa confiance et son estime entière, que d'être un peu versé dans la botanique. (P. 798 ; p. 180.$)$

La suite reprend, en plusieurs touches mais de plus en plus fermement, la défense des artistes. Le ton est de plus en plus assuré :

Berlioz est un artiste ; il est très pauvre, très brave et très fier. Peut-être bien a-t-il la scélératesse de penser en secret que tous les peuples de l'univers ne valent pas une gamme chromatique placée à propos, comme moi j'ai l'insolence de préférer une jacinthe blanche à la couronne de France. (P. 8IO ; p. 191.)

C'est dans cette même lettre que Sand se reproche de n'avoir pas toujours été assez artiste, contrainte par les circonstances de travailler trop vite. C'est là une manière très subtile de célébrer la grandeur de l’art : « [...] il y a quelque chose de vraiment noble et saint dans ce dévouement de l'artiste à son art, qui consiste à bien faire au prix de sa fortune, de sa gloire et de sa vie " (p. 8I2 ; p. 193). La huitième et dernière séquence est beaucoup plus agressive et ironique :

Écoute : si vous proclamez la république pendant mon absence, prenez tout ce qu'il y a chez moi, ne vous gênez pas ; j'ai des terres, donnez-les à ceux qui n'en ont pas ; [...] je demande qu'à mon retour on m'accorde une indemnité des pertes que $j$ 'aurais faites, savoir : une pipe, une plume et de l'encre ; moyennant quoi je gagnerai ma vie joyeusement, et passerai le reste de mes jours à écrire que vous avez bien fait. (P. 816 ; p. 197.)

S'agissant de la Lettre à Éverard, la date de l'écriture est très importante. En avril ı835, Michel de Bourges est à Paris et George Sand à Nohant. Elle rejoindra celui-ci dans la capitale au début du mois de mai. On lit entre les lignes l'envie de séduire et le désir d'avoir raison contre le Maître : avoir raison en tant qu'artiste contre l'homme de pouvoir, si génial soit-il. C'est l'éternel conflit entre le pouvoir qui rêve de tuer (faire " table rase ") et celui 
qui s'efforce de maintenir la vie. On retrouvera un débat assez semblable dans les lettres échangées avec Flaubert, entre l'absolutisme artistique de l'un, tempétueux et désespéré, et la vitalité prudente de l'autre, qu'on prend trop souvent pour du gâtisme. Après la sinistre traversée du Second Empire, malgré la guerre et la Commune, Sand a repris espoir, comme Nanon en témoigne.

Les dernières Lettres sont plus faciles à classer que celle qui est adressée à Éverard, comme une boucle qui se referme. De la septième, je ne retiendrai que le dénouement et la référence politique à l'attentat contre Louis-Philippe du 28 juillet I835, qui ramène définitivement l'écrivain sandien au cœur des affaires humaines. La suivante, intitulée "Le Prince ", poursuit dans cette voie en faisant une violente satire de Talleyrand. Retour à l'intimité dans les deux lettres suivantes, sur fond de mélancolie dans celle adressée à Jules Néraud, et sur un ton beaucoup plus léger dans celle "À Herbert " (Charles Didier), la seule où il soit vraiment question de voyage, de voyageurs et même de touristes. La lettre X est un hommage à Meyerbeer qui traite autant de religion que de musique, faisant de Robert le Diable (I83I) une " œuvre catholique " (p. 922 ; p. 299), en opposition au protestantisme des Huguenots, créés à l'Opéra de Paris en février I836, alors que le texte de Sand est daté de septembre 1836. En fait, on retrouve l'opposition entre la foi et le doute, né de la liberté. Il ne faut donc pas négliger la dimension politique de ce texte : la virulence de l'anticléricalisme rappelle les pages sur « l'ennemi du pape » de la lettre III. George Sand a vu deux fois l'opéra de Meyerbeer : "J'adore Les Huguenots'", écrit-elle à Liszt le Is mai I836, mais c'est en évoquant Berlioz qu'elle termine la lettre XI.

La lettre XII adressée à Nisard, très courte, est un véritable manifeste en faveur d'une littérature engagée dans le présent, y compris sur la question du mariage, qu'on lui a si souvent reproché, à cette époque surtout, de traiter de manière trop saint-simonienne. C'est pourquoi l'opposition entre réalisme et idéalisme, introduite par Zola et souvent reprise depuis, me paraît très impropre à décrire la spécificité de l'imaginaire sandien, dans lequel l'envol de l'oiseau va de pair avec la pesanteur du marcheur fatigué. L'hommage que Zola rend à Sand en 1876 contient beaucoup de haine refoulée, ou du moins un colossal mépris. Il constitue en tout cas un verdict de mort symbolique, une mise à l'écart définitive de Sand de la filiation littéraire des romanciers français ${ }^{6}$. Aujourd'hui, la critique sandienne s'intéresse davantage aux pro-

5. G. Sand, Correspondance, op. cit., vol. III, p. 370.

6. " George Sand toute sa vie a souhaité d'être un guérisseur, un ouvrier du progrès, l'apôtre d'une existence de béatitude. Elle était d'une nature poétique, ne pouvait marcher longtemps à terre, s'envolait au moindre souffle de l'inspiration. De là, l'étrange humanité qu'elle a 
blèmes d'énonciation et on devrait également pouvoir parler, entre autres sujets, du maniement sandien de l'ironie. Celui-ci, sans doute plus présent qu'on ne le dit dans l'ensemble de l'œuvre, se manifeste avec beaucoup d'évidence dans la lettre XII.

La réévaluation de l'écrivaine est loin d'être acquise. Beaucoup de textes sandiens mériteraient d'être relus sans préjugés et en se méfiant des interprétations au premier degré. Ne serait-ce que comme hypothèse de travail ouvrant de nouvelles pistes, il serait intéressant de lire Sand en se disant qu'elle ne pense jamais le mal qu'elle peut dire d'elle-même et de ses œuvres. Un corpus constitué par les Lettres d'un voyageur, Histoire de ma vie et la Correspondance Sand-Flaubert constituerait sans doute une bonne base de départ.

rêvée. Elle déformait toutes les réalités qu'elle touchait. Elle a créé un monde imaginaire, meilleur que le nôtre au point de vue de la justice absolue, un monde qu'on doit parcourir les yeux fermés, et qui prend alors le charme et la sympathie attendrie d'une vision évoquée par une bonne âme. " E. Zola, Écrits sur le roman, anthologie établie, présentée et annotée par H. Mitterand, Paris, Le Livre de Poche « Références », 2004, p. I69. 